\title{
On convergence theorems for single-valued and multi-valued mappings in $p$-uniformly convex metric spaces
}

\author{
JENJIRA PUiWONG and SATIT SAEJUNG
}

\begin{abstract}
We prove $\Delta$-convergence and strong convergence theorems of an iterative sequence generated by the Ishikawa's method to a fixed point of a single-valued quasi-nonexpansive mappings in $p$-uniformly convex metric spaces without assuming the metric convexity assumption. As a consequence of our single-valued version, we obtain a result for multi-valued mappings by showing that every multi-valued quasi-nonexpansive mapping taking compact values admits a quasi-nonexpansive selection whose fixed-point set of the selection is equal to the strict fixed-point set of the multi-valued mapping. In particular, we immediately obtain all of the convergence theorems of Laokul and Panyanak [Laokul, T.; Panyanak, B. A generalization of the $(C N)$ inequality and its applications. Carpathian J. Math. 36 (2020), no. 1, 81-90] and we show that some of their assumptions are superfluous.
\end{abstract}

\section{INTRODUCTION}

Suppose that $X:=(X, d)$ is a metric space. Denote by $2^{X}$ the set of all nonempty subsets of $X$. For a given multi-valued mapping $\boldsymbol{T}: X \rightarrow 2^{X}$, we say that $T: X \rightarrow X$ is a selection of $\boldsymbol{T}$ if $T x \in \boldsymbol{T} x$ for all $x \in X$. In this paper, we are interested in the following two notion which play an important role in analysis : a point $q \in X$ is called a fixed point (a strict fixed point, respectively) of a multi-valued mapping $\boldsymbol{T}$ if $q \in \boldsymbol{T} q(\{q\}=\boldsymbol{T} q$, respectively). Denote by $\operatorname{Fix}(\boldsymbol{T})$ and $\operatorname{SFix}(\boldsymbol{T})$ the sets of all fixed points and of strict fixed points of the multi-valued mapping $\boldsymbol{T}$, respectively. For more details, we refer the reader to the book of Rus et al. [20]. To simplify the notations for a single-valued mapping $T: X \rightarrow X$ we also write $\operatorname{Fix}(T):=\{q \in X: q=T q\}$ for the set of all fixed points of $T$. It is obvious that every single-valued mapping can be regarded as a multi-valued one. It follows that the notion of fixed point and of strict fixed point are the same when we are dealing with single-valued mappings.

The starting point of this paper is the following result proposed Laokul and Panyanak [12]. The relevant definitions will be given in the next section. The statements (a), (b), and (c) below are just Theorems 3.3, 3.4, and 3.5 of [12], respectively.

Theorem LP. Suppose that $X:=(X, d)$ is a complete 2-uniformly convex hyperbolic space. Suppose that $\boldsymbol{T}: X \rightarrow \mathcal{K}(X)$ is a Suzuki's generalized nonexpansive mapping such that $\operatorname{SFix}(\boldsymbol{T}) \neq$

Received: 29.10.2020. In revised form: 18.03.2021. Accepted: 25.03.2021

2010 Mathematics Subject Classification. 47H04, 47H09, 47H10.

Key words and phrases. Fixed point, strict fixed point, single-valued mapping, multi-valued mapping, p-uniformly convex metric space, selection of a multi-valued mapping, $\Delta$-convergence, strong convergence.

Corresponding author: Satit Saejung; saejung@kku.ac.th 
$\varnothing$. Suppose that $\left\{\alpha_{n}\right\},\left\{\beta_{n}\right\} \subset[0,1]$ and $\left\{z_{n}\right\} \subset X$ is iteratively defined as follows:

$z_{1} \in X$ is arbitrarily chosen;

$z_{n}^{\prime} \in \boldsymbol{T} z_{n}$ such that $d\left(z_{n}, z_{n}^{\prime}\right)=\sup \left\{d\left(z_{n}, z\right): z \in \boldsymbol{T} z_{n}\right\}$

$w_{n}:=\left(1-\beta_{n}\right) z_{n} \oplus \beta_{n} z_{n}^{\prime}$;

$w_{n}^{\prime} \in \boldsymbol{T} w_{n}$ such that $d\left(w_{n}, w_{n}^{\prime}\right)=\sup \left\{d\left(w_{n}, w\right): w \in \boldsymbol{T} w_{n}\right\}$;

$z_{n+1}:=\left(1-\alpha_{n}\right) z_{n} \oplus \alpha_{n} w_{n}^{\prime}$ for each $n \geq 1$.

The following statements are true.

(a) If $\alpha_{n}, \beta_{n} \in[a, b] \subset(0,1)$ for all $n \geq 1$, then $\left\{z_{n}\right\} \Delta$-converges to a strict fixed point of $\boldsymbol{T}$.

(b) If $\boldsymbol{T}$ satisfies Condition $I^{*}$ and $\alpha_{n}, \beta_{n} \in[a, b] \subset(0,1)$ for all $n \geq 1$, then $\left\{z_{n}\right\}$ converges strongly to a strict fixed point of $\boldsymbol{T}$.

(c) If $\boldsymbol{T}$ is semicompact ${ }^{*}$ and $\sum_{n} \alpha_{n} \beta_{n}=\infty$ and $\lim _{n} \beta_{n}=0$, then $\left\{z_{n}\right\}$ converges strongly to a strict fixed point of $\boldsymbol{T}$.

Recall that a metric space $X:=(X, d)$ is a 2-uniformly convex hyperbolic space if there exist a function $W: X \times X \times[0,1] \rightarrow X$ and a constant $C>0$ such that the following conditions are satisfied by all elements $x, y, z, w \in X$ and all real numbers $s, t \in[0,1]$ :

(W1) $d(W(x, y, t), z) \leq(1-t) d(x, z)+t d(y, z)$;

(W2) $d(W(x, y, s), W(x, y, t))=|s-t| d(x, y)$;

(W3) $W(x, y, s)=W(y, x, 1-s)$;

(W4) $d(W(x, y, s), W(z, w, s)) \leq(1-s) d(x, z)+s d(y, w)$;

(W5) $d^{2}(W(x, y, 1 / 2), z)+C d^{2}(x, y) \leq \frac{1}{2} d^{2}(x, z)+\frac{1}{2} d^{2}(y, z)$.

Laokul and Panyanak [12] claimed that every uniformly convex Banach space and every $\operatorname{CAT}(\kappa)$ space with a small diameter where $\kappa>0$ are 2-uniformly convex hyperbolic spaces (see [12, Remark 1.1]). We note that the claim above is not correct. First, not every uniformly convex Banach space is a 2-uniformly convex hyperbolic space. In fact, every inner product space and every bounded convex subset of a uniformly convex Banach space are 2-uniformly convex hyperbolic spaces. The boundedness cannot be omitted in the latter spaces, for more detail, see [26]. Secondly, it is easy to see that a certain subset of $\mathbb{S}^{2}:=\left\{\left(s_{1}, s_{2}, s_{3}\right) \in \mathbb{R}^{3}: s_{1}^{2}+s_{2}^{2}+s_{3}^{2}=1\right\}$ with the spherical distance $d$ which is a CAT(1) space together with a function $W$ defined by the natural geodesic structure does not satisfy Condition (W4). In fact, let $x=z:=(0,0,1), y:=(\sqrt{3} / 2,0,1 / 2), w:=$ $(0, \sqrt{3} / 2,1 / 2)$, and $s=1 / 2$. It follows that $d(W(x, y, s), W(z, w, s))=\arccos (3 / 4) \approx 0.723$ but $(1-s) d(x, z)+s d(y, w)=\frac{1}{2} \arccos (1 / 4) \approx 0.659$.

For the argument above, it is our purpose to use a notion of $p$-uniformly convex metric spaces of Naor and Silberman [15], which is more general than the one in [12], to reestablish their results in a more general setting with a simpler proof. Our result does not require Conditions (W1) and (W4). We also show that there are mappings such that they are applicable to our results but beyond the scope of [12].

\section{MAIN RESULTS}

Let $X:=(X, d)$ be a metric space and $x, y \in X$. A continuous map $\gamma:[0,1] \rightarrow X$ is called a geodesic joining $x$ and $y$ if $\gamma(0)=x, \gamma(1)=y$ and

$$
d(\gamma(s), \gamma(t))=|s-t| d(x, y) \text { for all } s, t \in[0,1] .
$$

A metric space $X:=(X, d)$ is said to be a geodesic metric space if for any two points in $X$ there exists a geodesic $\gamma$ joining them. If for any two points in $X$ are joined by a unique geodesic, then we say that $X$ is a uniquely geodesic metric space. 
A geodesic metric space $(X, d)$ is called p-uniformly convex [15] where $2 \leq p<\infty$ if there exists a constant $C:=C_{X} \in(0,1)$ such that for any $x, y, z \in X$ and for any geodesic $\gamma:[0,1] \rightarrow X$ joining $x$ and $y$, the following inequality holds:

$$
d^{p}(\gamma(t), z) \leq(1-t) d^{p}(x, z)+t d^{p}(y, z)-C t(1-t) d^{p}(x, y) \text { for all } t \in[0,1] .
$$

Note that the inequality above can hold only for $p \geq 2$ (see [15]) so this is why we assume such a restriction. It is obvious that this notion includes every 2-uniformly convex hyperbolic space. Moreover, the inequality proved in Theorem 2.2 of [12] is also included in the preceding inequality. It is worth mentioning that Conditions (W1) and (W4) are not required in this setting as were the cases in [12].

Lemma 2.1. Every p-uniformly convex geodesic metric space is uniquely geodesic, that is, if $X$ is a p-uniformly convex geodesic metric space, then for each $x, y \in X$ there exists a unique geodesic joining $x$ and $y$.

Proof. We follow the proof of Lemma 2.2 of [16] which is stated for the case $p=2$. Suppose that $x, y$ are two elements in a $p$-uniformly convex geodesic metric space $X:=(X, d)$ and suppose that $\gamma_{1}$ and $\gamma_{2}$ are two geodesic joining $x$ and $y$. We show that $\gamma_{1}(t)=\gamma_{2}(t)$ for all $t \in(0,1)$. To see this, let $t \in(0,1)$ and let $\eta$ be a geodesic joining $\gamma_{1}(t)$ and $\gamma_{2}(t)$. We observe that

$$
\begin{aligned}
d^{p}(x, \eta(1 / 2)) & \leq \frac{1}{2} d^{p}\left(x, \gamma_{1}(t)\right)+\frac{1}{2} d^{p}\left(x, \gamma_{2}(t)\right)-\frac{C}{4} d^{p}\left(\gamma_{1}(t), \gamma_{2}(t)\right) \\
& =\frac{1}{2} d^{p}\left(\gamma_{1}(0), \gamma_{1}(t)\right)+\frac{1}{2} d^{p}\left(\gamma_{2}(0), \gamma_{2}(t)\right)-\frac{C}{4} d^{p}\left(\gamma_{1}(t), \gamma_{2}(t)\right) \\
& =t^{p} d^{p}(x, y)-\frac{C}{4} d^{p}\left(\gamma_{1}(t), \gamma_{2}(t)\right) .
\end{aligned}
$$

Similarly, we also have

$$
d^{p}(y, \eta(1 / 2)) \leq(1-t)^{p} d^{p}(x, y)-\frac{C}{4} d^{p}\left(\gamma_{1}(t), \gamma_{2}(t)\right) .
$$

In particular,

$$
\begin{aligned}
d(x, y) & \leq d(x, \eta(1 / 2))+d(y, \eta(1 / 2)) \\
& \leq\left(t^{p} d^{p}(x, y)-\frac{C}{4} d^{p}\left(\gamma_{1}(t), \gamma_{2}(t)\right)\right)^{1 / p}+\left((1-t)^{p} d^{p}(x, y)-\frac{C}{4} d^{p}\left(\gamma_{1}(t), \gamma_{2}(t)\right)\right)^{1 / p} \\
& \leq t d(x, y)+(1-t) d(x, y)=d(x, y) .
\end{aligned}
$$

This implies that $\gamma_{1}(t)=\gamma_{2}(t)$ and the proof is finished.

If $X$ is a uniquely geodesic space, (for example, $X$ is a $p$-uniformly convex geodesic metric space), then we write

$$
(1-t) x \oplus t y:=\gamma(t)
$$

where $\gamma$ is the unique geodesic joining $x$ and $y$. In particular, for a uniquely geodesic space $X$, we also have $(1-t) x \oplus t y=t y \oplus(1-t) x$ for all $x, y \in X$ and $t \in[0,1]$.

Recall a mapping $f: X \rightarrow \mathbb{R}$ is convex if

$$
f((1-t) x \oplus t y) \leq(1-t) f(x)+t f(y) \text { for all } t \in[0,1] \text { and } x, y \in X .
$$

It is clear that if $X:=(X, d)$ is $p$-uniformly convex, then $f(x):=d^{p}(x, z)$ is convex for a fixed element $z \in X$. 
2.1. $\Delta$-convergence and metric projection. We now discuss the concept of $\Delta$-convergence introduced by $\operatorname{Lim}$ [13]. Let $\left\{x_{n}\right\}$ be a bounded sequence in $X$. The asymptotic center of $\left\{x_{n}\right\}$ is defined by

$$
A\left(\left\{x_{n}\right\}\right):=\left\{x \in X: \limsup _{n} d\left(x, x_{n}\right)=\inf _{y \in X} \limsup _{n} d\left(y, x_{n}\right)\right\} .
$$

We say that $\left\{x_{n}\right\}$ is $\Delta$-convergent to $x \in X$, denoted by $x_{n} \stackrel{\Delta}{\rightarrow} x$, if $A\left(\left\{x_{n_{k}}\right\}\right)=\{x\}$ for all subsequences $\left\{x_{n_{k}}\right\}$ of $\left\{x_{n}\right\}$. The asymptotic center of a bounded sequence in a complete $p$-uniformly convex geodesic metric space is a singleton as shown below. The second assertion is related to [26, lemma 2].

Theorem 2.1. If $\left\{x_{n}\right\}$ is a bounded sequence in a complete p-uniformly convex geodesic metric space $X:=(X, d)$, then $A\left(\left\{x_{n}\right\}\right)=\{z\}$ for some $z \in X$. Furthermore, if $X$ is $p$-uniformly convex with a constant $C$, then

$$
\limsup _{n} d^{p}\left(z, x_{n}\right)+C d^{p}(y, z) \leq \underset{n}{\limsup } d^{p}\left(y, x_{n}\right)
$$

for all $y \in X$.

Proof. Suppose that $\left\{x_{n}\right\}$ is a bounded sequence in a complete $p$-uniformly convex geodesic metric space $X:=(X, d)$. Define $f(x):=\limsup _{n} d^{p}\left(x, x_{n}\right)$ for all $x \in X$. It is clear that $f$ is convex and continuous. Put $\alpha:=\inf _{y \in X} f(y)$. Choose a sequence $\left\{y_{m}\right\}$ in $X$ such that $\alpha \leq f\left(y_{m}\right) \leq \alpha+\frac{1}{m}$ for all $m \geq 1$. Let us consider the following:

$$
\begin{aligned}
\alpha & \leq f\left(\frac{1}{2} y_{m} \oplus \frac{1}{2} y_{k}\right) \\
& =\limsup _{n} d^{p}\left(\frac{1}{2} y_{m} \oplus \frac{1}{2} y_{k}, x_{n}\right) \\
& \leq \frac{1}{2} \limsup _{n} d^{p}\left(y_{m}, x_{n}\right)+\frac{1}{2} \limsup _{n} d^{p}\left(y_{k}, x_{n}\right)-\frac{C}{4} d^{p}\left(y_{m}, y_{k}\right) \\
& =\frac{1}{2} f\left(y_{m}\right)+\frac{1}{2} f\left(y_{k}\right)-\frac{C}{4} d^{p}\left(y_{m}, y_{k}\right) \\
& \leq \frac{1}{2}\left(\alpha+\frac{1}{m}\right)+\frac{1}{2}\left(\alpha+\frac{1}{k}\right)-\frac{C}{4} d^{p}\left(y_{m}, y_{k}\right) .
\end{aligned}
$$

It follows that $\lim _{m, k} d^{p}\left(y_{m}, y_{k}\right)=0$. Then $\left\{y_{m}\right\}$ is a Cauchy sequence in $X$. In particular, since $X$ is complete, the sequence $\left\{y_{m}\right\}$ converges to some element $z$ in $X$. Since $f$ is continuous, we have $f(z)=\lim _{m} f\left(y_{m}\right)=\alpha$. Hence $z \in A\left(\left\{x_{n}\right\}\right)$. Suppose that there exists $z^{\prime}$ in $X$ such that an element $z^{\prime} \in A\left(\left\{x_{n}\right\}\right)$. Put $y_{1}^{\prime}=y_{3}^{\prime}=\cdots=z$ and $y_{2}^{\prime}=y_{4}^{\prime}=$ $\cdots=z^{\prime}$. Then $f\left(y_{m}^{\prime}\right)=\alpha$ for all $m \geq 1$. Using the same method as above, we have $\left\{y_{m}^{\prime}\right\}$ is Cauchy and hence $z^{\prime}=z$.

We now prove the second assertion. Let $y \in X$ and let $t \in(0,1)$. Note that

$$
d^{p}\left((1-t) y \oplus t z, x_{n}\right) \leq(1-t) d^{p}\left(y, x_{n}\right)+t d^{p}\left(z, x_{n}\right)-C t(1-t) d^{p}(y, z) .
$$

It follows that

$$
\begin{aligned}
f(z) & \leq f((1-t) y \oplus t z) \\
& \leq(1-t) f(y)+t f(z)-C(1-t) t d^{p}(y, z) .
\end{aligned}
$$

In particular, we have

$$
f(z) \leq f(y)-C t d^{p}(y, z)
$$

Letting $t \uparrow 1$ gives the desired result. This completes the proof. 
It is also known that every bounded sequence in a metric space has a regular subsequence (see [8]). Recall that $\left\{x_{n}\right\}$ is regular if

$$
\inf _{y \in X} \limsup _{k} d\left(y, x_{n_{k}}\right)=\inf _{y \in X} \limsup _{n} d\left(x, x_{n}\right)
$$

for all subsequences $\left\{x_{n_{k}}\right\}$ of $\left\{x_{n}\right\}$. In particular, if $X$ is a complete $p$-uniformly convex geodesic metric space, then every bounded sequence has a $\Delta$-convergent subsequence.

The proof of the following result is similar to that of Proposition 3.1 of [10]. Recall that $\omega_{\Delta}\left(\left\{x_{n}\right\}\right)$ denotes the set of $\Delta$-limits of all $\Delta$-convergent subsequences of $\left\{x_{n}\right\}$.

Theorem 2.2. Suppose that $X$ is a complete p-uniformly convex geodesic metric space $X$. If $\left\{x_{n}\right\}$ is a bounded sequence in $X$ such that $\lim _{n} d\left(x_{n}, q\right)$ exists for all $q \in \omega_{\Delta}\left(\left\{x_{n}\right\}\right)$, then $x_{n} \stackrel{\Delta}{\rightarrow} x$ for some $x \in X$.

Proof. Suppose that $A\left(\left\{x_{n}\right\}\right)=\{q\}$ and $A\left(\left\{x_{n_{k}}\right\}\right)=\left\{q^{\prime}\right\}$ where $\left\{x_{n_{k}}\right\}$ is a subsequence of $\left\{x_{n}\right\}$ and $q, q^{\prime} \in X$. We show that $q=q^{\prime}$. Since $\left\{x_{n_{k}}\right\}$ is bounded, there exists a subsequence $\left\{y_{k}\right\}$ of $\left\{x_{n_{k}}\right\}$ such that $y_{k} \stackrel{\Delta}{\rightarrow} y$ for some $y \in X$. By our assumption, $\lim _{n} d\left(x_{n}, y\right)$ exists. If $q \neq y$, then $\limsup _{n} d\left(x_{n}, q\right)<\lim _{n} d\left(x_{n}, y\right)=\lim _{k} d\left(y_{k}, y\right)<$ $\lim \sup _{k} d\left(y_{k}, q\right) \leq \lim \sup _{n} d\left(x_{n}, q\right)$ which is impossible. Hence $q=y$. If $q^{\prime} \neq y$, then $\lim \sup _{k} d\left(x_{n_{k}}, q^{\prime}\right)<\lim _{n} d\left(x_{n}, y\right)=\lim _{k} d\left(y_{k}, y\right)<\lim \sup _{k} d\left(y_{k}, q^{\prime}\right) \leq \lim \sup _{k} d\left(x_{n_{k}}, q^{\prime}\right)$ which is impossible. Hence $q^{\prime}=y$. This implies that $q=q^{\prime}$ and hence the proof is finished.

We discuss a concept of metric projection in this framework. Suppose that $D$ is a closed convex subset of a complete $p$-uniformly convex geodesic metric space $X$. Recall that a subset $D$ of $X$ is convex if $(1-t) x \oplus t y \in D$ for all $x, y \in D$ and $t \in[0,1]$. We also get the following characterization.

Following the proof of Theorem 2.1 with a slight modification, we also obtain the following result.

Theorem 2.3. Suppose that $D$ is a closed convex subset of a complete p-uniformly convex geodesic metric space $X$ with a constant $C$. For a given $x \in X$, there exists a unique element $z \in D$ such that

Equivalently,

$$
d^{p}(z, x)=\inf _{y \in D} d^{p}(y, x)
$$

$$
d(z, x)=\inf _{y \in D} d(y, x) .
$$

In this case, we write $z:=\operatorname{Proj}_{D} x$. Furthermore, suppose that $x \in X$ and $z \in D$. Then $z=\operatorname{Proj}_{D} x$ if and only if

$$
d^{p}(x, z)+C d^{p}(z, y) \leq d^{p}(x, y) \text { for all } y \in D .
$$

2.2. Fejér monotone convergence principle. We prove the following convergence principle in $p$-uniformly convex geodesic metric spaces. Recall that $\left\{x_{n}\right\}$ is a Fejér sequence in a metric space $X:=(X, d)$ with respect to a subset $D$ of $X$ if $d\left(x_{n+1}, q\right) \leq d\left(x_{n}, q\right)$ for all $n \geq 1$ and $q \in D$.

Theorem 2.4. Suppose that $D$ is a closed convex subset of a complete p-uniformly convex geodesic metric space $X$ and $\left\{x_{n}\right\}$ is a Fejer sequence with respect to D. Then $\left\{\operatorname{Proj}_{D} x_{n}\right\}$ is a Cauchy sequence.

Proof. Put $u_{n}:=\operatorname{Proj}_{D} x_{n}$. For each $k \geq 1$, we note that $d\left(x_{n+k}, u_{n}\right) \leq d\left(x_{n}, u_{n}\right)$. Moreover, it follows from Theorem 2.3 that

$$
d^{p}\left(x_{n+k}, u_{n+k}\right)+C d^{p}\left(u_{n+k}, u_{n}\right) \leq d^{p}\left(x_{n+k}, u_{n}\right) \leq d^{p}\left(x_{n}, u_{n}\right) .
$$


In particular, we have $d^{p}\left(x_{n+1}, u_{n+1}\right) \leq d^{p}\left(x_{n}, u_{n}\right)$ and hence $\lim _{n} d^{p}\left(x_{n}, u_{n}\right)$ exists. It follows that $\lim _{n, k} d^{p}\left(u_{n+k}, u_{n}\right)=0$, that is, $\left\{u_{n}\right\}$ is a Cauchy sequence.

2.3. Convergence theorems. We are ready to discuss the convergence of the following two iterative sequences. Suppose that $X:=(X, d)$ is a $p$-uniformly convex geodesic metric space and $T: X \rightarrow X$ is a mapping. Let $\left\{x_{n}\right\}$ be iteratively defined as follows: $x_{1} \in X$ is arbitrarily chosen and for each $n \geq 1$

$$
\begin{aligned}
y_{n} & :=\left(1-\beta_{n}\right) x_{n} \oplus \beta_{n} T x_{n} ; \\
x_{n+1} & :=\left(1-\alpha_{n}\right) x_{n} \oplus \alpha_{n} T y_{n} ;
\end{aligned}
$$

where $\left\{\alpha_{n}\right\}$ and $\left\{\beta_{n}\right\}$ are sequences in $[0,1]$. This scheme is known as the Ishikawa's iteration ([9]). If $\beta_{n}:=0$ for all $n \geq 1$, then the Ishikawa iteration becomes the Mann's iteration ([14]).

We now give some remark on the Ishikawa's and Mann's iterations as suggested by the referee. It can be seen that the Ishikawa's iteration is complicated than the Mann's. The Ishikawa's iteration was proposed the first time to deal with the problem of finding a fixed point of Lipschitz pseudocontractions. Moreover, it was proved by Chidume and Mutangadura [4] that the simpler Mann's iteration cannot guarantee the convergence of the iteration to a fixed point of Lipschitz pseudocontractions. To our best knowledge, there is no result showing that the Ishikawa's iteration (Mann's iteration, resp.) is convergent while the Mann's (Kransnoselskii's, resp.) is not in the setting of quasi-nonexpansive mappings. The only one reason we discuss the Ishikawa's iteration here is just to recover the main results of [12] (see Theorem LP).

Recall that a mapping $T: X \rightarrow X$ is quasi-nonexpansive if $\operatorname{Fix}(T):=\{q \in X: q=T q\}$ is nonempty and $d(T x, q) \leq d(x, q)$ for all $x \in X$ and $q \in \operatorname{Fix}(T)$. We say that $T$ is $\Delta$-demiclosed if $q \in \operatorname{Fix}(T)$ whenever $\left\{x_{n}\right\}$ is a sequence in $X$ such that $x_{n} \stackrel{\Delta}{\rightarrow} q$ and $\lim _{n} d\left(x_{n}, T x_{n}\right)=0$.

Lemma 2.2. Suppose that $X:=(X, d)$ is a p-uniformly convex geodesic metric space and $T$ : $X \rightarrow X$ is a quasi-nonexpansive mapping. Then $\operatorname{Fix}(T)$ is closed and convex.

Proof. Suppose that $X$ is a $p$-uniformly convex with a constant $C:=C_{X}$. We first show that $\operatorname{Fix}(T)$ is closed. To see this, let $\left\{q_{n}\right\}$ be a sequence in $\operatorname{Fix}(T)$ such that $\lim _{n} q_{n}=q$ for some $q \in X$. Note that $d\left(T q, q_{n}\right) \leq d\left(q, q_{n}\right)$. It follows that $\lim _{n} q_{n}=T q$ and hence $q=T q$, that is, $q \in \operatorname{Fix}(T)$. Finally, we show that $\operatorname{Fix}(T)$ is convex. Suppose that $q_{1}, q_{2} \in \operatorname{Fix}(T)$ and $t \in[0,1]$. To see that $q:=(1-t) q_{1} \oplus t q_{2} \in \operatorname{Fix}(T)$, we note that

$$
d\left(T q, q_{1}\right) \leq d\left(q, q_{1}\right)=t d\left(q_{1}, q_{2}\right) \text { and } d\left(T q, q_{2}\right) \leq(1-t) d\left(q_{1}, q_{2}\right) .
$$

This implies that

$$
d\left(q_{1}, q_{2}\right) \leq d\left(T q, q_{1}\right)+d\left(T q, q_{2}\right) \leq t d\left(q_{1}, q_{2}\right)+(1-t) d\left(q_{1}, q_{2}\right)=d\left(q_{1}, q_{2}\right) .
$$

In particular, we have

$$
d\left(T q, q_{1}\right)=t d\left(q_{1}, q_{2}\right) \quad \text { and } \quad d\left(T q, q_{2}\right)=(1-t) d\left(q_{1}, q_{2}\right) .
$$

Since there exists a unique geodesic joining $q_{1}$ and $q_{2}$, we have

$$
T q=(1-t) q_{1} \oplus t q_{2}=q .
$$

That is, $q \in \operatorname{Fix}(T)$.

Using the Ishikawa iteration, we obtain the following $\Delta$-convergence theorem. It is worth mentioning that we can prove that the $\Delta$-limit of the iterative sequence is the strong-limit of the sequence of its projection on the fixed point set of the mapping. 
Theorem 2.5. Suppose that $X:=(X, d)$ is a complete p-uniformly convex geodesic metric space and $T: X \rightarrow X$ is a quasi-nonexpansive mapping which is $\Delta$-demiclosed. Suppose that $\left\{x_{n}\right\}$ is generated the Ishikawa iteration where $\left\{\alpha_{n}\right\}$ and $\left\{\beta_{n}\right\}$ satisfy one of the following conditions:

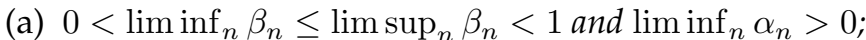

(b) $0<\liminf _{n} \alpha_{n} \leq \limsup _{n} \alpha_{n}<1$ and $\beta_{n}=0$ for all $n \geq 1$.

Then $x_{n} \stackrel{\Delta}{\rightarrow} q \in \operatorname{Fix}(T)$ and $q=\lim _{n} \operatorname{Proj}_{\mathrm{Fix}(T)} x_{n}$.

Proof. Suppose that $X$ is a $p$-uniformly convex with a constant $C:=C_{X}$. Let $q \in \operatorname{Fix}(T)$. For each $n \geq 1$, we have

$$
\begin{aligned}
d^{p}\left(y_{n}, q\right) & =d^{p}\left(\left(1-\beta_{n}\right) x_{n} \oplus \beta_{n} T x_{n}, q\right) \\
& \leq\left(1-\beta_{n}\right) d^{p}\left(x_{n}, q\right)+\beta_{n} d^{p}\left(T x_{n}, q\right)-C \beta_{n}\left(1-\beta_{n}\right) d^{p}\left(x_{n}, T x_{n}\right) \\
& \leq\left(1-\beta_{n}\right) d^{p}\left(x_{n}, q\right)+\beta_{n} d^{p}\left(x_{n}, q\right)-C \beta_{n}\left(1-\beta_{n}\right) d^{p}\left(x_{n}, T x_{n}\right) \\
& =d^{p}\left(x_{n}, q\right)-C \beta_{n}\left(1-\beta_{n}\right) d^{p}\left(x_{n}, T x_{n}\right) .
\end{aligned}
$$

Then

$$
\begin{aligned}
d^{p}\left(x_{n+1}, q\right)= & d^{p}\left(\left(1-\alpha_{n}\right) x_{n} \oplus \alpha_{n} T y_{n}, q\right) \\
\leq & \left(1-\alpha_{n}\right) d^{p}\left(x_{n}, q\right)+\alpha_{n} d^{p}\left(T y_{n}, q\right)-C \alpha_{n}\left(1-\alpha_{n}\right) d^{p}\left(x_{n}, T y_{n}\right) \\
\leq & \left(1-\alpha_{n}\right) d^{p}\left(x_{n}, q\right)+\alpha_{n} d^{p}\left(y_{n}, q\right)-C \alpha_{n}\left(1-\alpha_{n}\right) d^{p}\left(x_{n}, T y_{n}\right) \\
\leq & \left(1-\alpha_{n}\right) d^{p}\left(x_{n}, q\right)+\alpha_{n} d^{p}\left(x_{n}, q\right)-C \alpha_{n} \beta_{n}\left(1-\beta_{n}\right) d^{p}\left(x_{n}, T x_{n}\right) \\
& \quad-C \alpha_{n}\left(1-\alpha_{n}\right) d^{p}\left(x_{n}, T y_{n}\right) .
\end{aligned}
$$

In particular, we have $d\left(x_{n+1}, q\right) \leq d\left(x_{n}, q\right)$, that is, $\left\{x_{n}\right\}$ is a Fejér sequence with respect to $\operatorname{Fix}(T)$ and hence $\lim _{n} d^{p}\left(x_{n}, q\right)$ exists. It follows that $\sum_{n} \alpha_{n} \beta_{n}\left(1-\beta_{n}\right) d^{p}\left(x_{n}, T x_{n}\right)<\infty$ and $\sum_{n} \alpha_{n}\left(1-\alpha_{n}\right) d^{p}\left(x_{n}, T y_{n}\right)<\infty$. Suppose that either (a) or (b) is satisfied. This implies that $\sum_{n} d^{p}\left(x_{n}, T x_{n}\right)<\infty$ and hence $\lim _{n} d\left(x_{n}, T x_{n}\right)=0$.

We now prove that $x_{n} \stackrel{\Delta}{\rightarrow} q \in \operatorname{Fix}(T)$. Note that $\lim _{n} d\left(x_{n}, q^{\prime}\right)$ exists for all $q^{\prime} \in \operatorname{Fix}(T)$. It suffices to show that $\omega_{\Delta}\left(\left\{x_{n}\right\}\right) \subset \operatorname{Fix}(T)$. To see this, let $\left\{x_{n_{k}}\right\}$ be a subsequence of $\left\{x_{n}\right\}$ such that $x_{n_{k}} \stackrel{\Delta}{\rightarrow} q$ for some $q \in X$. It follows from the $\Delta$-demiclosedness of $T$ and $\lim _{k} d\left(x_{n_{k}}, T x_{n_{k}}\right)=0$ that $q \in \operatorname{Fix}(T)$. It follows from Theorem 2.2 that $x_{n} \stackrel{\Delta}{\rightarrow} q$ for some $q \in X$. It follows from the $\Delta$-demiclosedness of $T$ and $\lim _{n} d\left(x_{n}, T x_{n}\right)=0$ that $q \in \operatorname{Fix}(T)$.

Finally, we show that $\lim _{n} \operatorname{Proj}_{\mathrm{Fix}(T)} x_{n}=q$. Since $\operatorname{Fix}(T)$ is closed and convex (by Lemma 2.2), the projection $\operatorname{Proj}_{\mathrm{Fix}(T)}$ is well-defined by Theorem 2.3. Let $u_{n}:=\operatorname{Proj}_{\mathrm{Fix}(T)} x_{n}$. It follows from Theorem 2.4 that $\left\{u_{n}\right\}$ is a Cauchy sequence in $\operatorname{Fix}(T)$. Hence $\lim _{n} u_{n}=u$ for some $u \in \operatorname{Fix}(T)$ because $\operatorname{Fix}(T)$ is closed. Furthermore, it follows from Theorem 2.3 that

This implies that

$$
d^{p}\left(x_{n}, u_{n}\right) \leq d^{p}\left(x_{n}, u_{n}\right)+C d^{p}\left(u_{n}, q\right) \leq d^{p}\left(x_{n}, q\right) .
$$

$$
\limsup _{n} d\left(x_{n}, u\right) \leq \limsup _{n} d\left(x_{n}, q\right) .
$$

It follows from $x_{n} \stackrel{\Delta}{\rightarrow} q$ that $\lim _{n} u_{n}=u=q$ and this completes the proof.

To discuss the strong convergence, we recall the following concept.

Definition 2.1 ([22]). Suppose that $X:=(X, d)$ is a metric space. A mapping $T: X \rightarrow X$

- satisfies Condition I if $\operatorname{Fix}(T) \neq \varnothing$ and there exists a nondecreasing function $h$ : $[0, \infty) \rightarrow[0, \infty)$ such that $h$ vanishes only at zero and

$$
h(\operatorname{dist}(x, \operatorname{Fix}(T))) \leq d(x, T x) \text { for all } x \in X ;
$$


- is semicompact if every sequence $\left\{x_{n}\right\}$ in $X$ with $\lim _{n} d\left(x_{n}, T x_{n}\right)=0$ contains a convergent subsequence;

- is strongly demiclosed if $q \in \operatorname{Fix}(T)$ whenever $\left\{x_{n}\right\}$ is a sequence in $X$ such that $\lim _{n} d\left(x_{n}, T x_{n}\right)=0$ and $\lim _{n} x_{n}=q \in X$.

Here $\operatorname{dist}(x, F):=\inf \{d(x, a): a \in F\}$.

Using the preceding notion and the proof of Theorem 2.5, we obtain the following strong convergence theorem.

Theorem 2.6. Suppose that $X, d, T$ are the same as Theorem 2.5. Suppose that $\left\{x_{n}\right\}$ is generated the Ishikawa iteration where $\left\{\alpha_{n}\right\}$ and $\left\{\beta_{n}\right\}$ satisfy one of the following conditions:

(a) $\sum_{n} \alpha_{n} \beta_{n}\left(1-\beta_{n}\right)=\infty$;

(b) $\beta_{n}=0$ for all $n \geq 1$ and $\sum_{n} \alpha_{n}\left(1-\alpha_{n}\right)=\infty$.

If one of the following conditions is satisfied:

(i) T satisfies Condition I;

(ii) $T$ is semicompact and strongly demiclosed,

then $\left\{x_{n}\right\}$ converges strongly to a fixed point of $T$.

To prove this result, let us recall the following fact from [21].

Lemma 2.3. Suppose that $F$ is a closed subset of a metric space $X:=(X, d)$ and suppose that $\left\{x_{n}\right\}$ is a Fejér sequence with respect to $F$. Then $\left\{x_{n}\right\}$ converges strongly to a point in $F$ if and only if $\lim \inf _{n} \operatorname{dist}\left(x_{n}, F\right)=0$.

Lemma 2.4. If $\left\{\alpha_{n}\right\}$ and $\left\{\gamma_{n}\right\}$ are two sequences of nonnegative real numbers such that $\sum_{n} \alpha_{n}=$ $\infty$ and $\sum_{n} \alpha_{n} \gamma_{n}<\infty$, then $\liminf _{n} \gamma_{n}=0$.

Proof of Theorem 2.6. It follows from the proof of Theorem 2.5 that

$$
\sum_{n} \alpha_{n} \beta_{n}\left(1-\beta_{n}\right) d^{p}\left(x_{n}, T x_{n}\right)<\infty \quad \text { and } \quad \sum_{n} \alpha_{n}\left(1-\alpha_{n}\right) d^{p}\left(x_{n}, T y_{n}\right)<\infty
$$

Consequently, it follows from Lemma 2.4 and either (a) or (b) that $\lim \inf _{n} d\left(x_{n}, T x_{n}\right)=0$. We consider the following two cases.

Case 1: $T$ satisfies Condition I. Note that $\operatorname{Fix}(T)$ is closed and $\left\{x_{n}\right\}$ is a Fejér sequence with respect to $\operatorname{Fix}(T)$. It suffices to prove that $\lim \inf _{n} \operatorname{dist}\left(x_{n}, \operatorname{Fix}(T)\right)=0$. Note that there exists a nondecreasing function $h:[0, \infty) \rightarrow[0, \infty)$ such that $h$ vanishes only at zero and

$$
h\left(\operatorname{dist}\left(x_{n}, \operatorname{Fix}(T)\right)\right) \leq d\left(x_{n}, T x_{n}\right) \text { for all } n \geq 1 .
$$

In particular, we have $\liminf \operatorname{in}_{n} h\left(\operatorname{dist}\left(x_{n}, \operatorname{Fix}(T)\right)\right)=0$. By the property of the function $h$, we can conclude that $\liminf { }_{n} \operatorname{dist}\left(x_{n}, \operatorname{Fix}(T)\right)=0$. The conclusion follows from Lemma 2.3.

Case 2: $T$ is semicompact and strongly demiclosed. It follows from $\lim \inf _{n} d\left(x_{n}, T x_{n}\right)=$ 0 that there exists a convergent subsequence $\left\{x_{n_{k}}\right\}$ of $\left\{x_{n}\right\}$. We assume that $\lim _{k} x_{n_{k}}=q$ for some $q \in X$. Since $T$ is strongly demiclosed, we have $q \in \operatorname{Fix}(T)$. In particular, since $\lim _{n} d\left(x_{n}, q\right)$ exists and $\lim _{k} d\left(x_{n_{k}}, q\right)=0$, we conclude that $\lim _{n} d\left(x_{n}, q\right)=0$. The proof is finished.

2.4. Multi-valued mappings and Theorem LP. To formulate the problem for multi-valued mappings, we recall the following notion. In this part, we use the bold symbol to denote a multi-valued mapping and the normal one for a single-valued mapping. Suppose that 
$X:=(X, d)$ is a metric space. Denote by $\mathcal{K}(X)(\mathcal{B C}(X)$, respectively) the set of all nonempty compact subsets (nonempty bounded and closed subsets, respectively) of $X$. Let $\mathcal{H}$ be the Pompeiu-Hausdorff distance on $\mathcal{B C}(X)$, that is, for $A, B \in \mathcal{B C}(X)$

$$
\mathcal{H}(A, B):=\max \left\{\sup _{a \in A} \operatorname{dist}(a, B), \sup _{b \in B} \operatorname{dist}(b, A)\right\} .
$$

Note that $\mathcal{H}(\{x\}, C)=\sup \{d(x, c): c \in C\}$ for each $x \in X$ and $C \in \mathcal{B C}(X)$. It is clear that $(\mathcal{B C}(X), \mathcal{H})$ becomes a metric space and so does the subspace $(\mathcal{K}(X), \mathcal{H})$. For more details on the role of the Pompeiu-Hausdorff distance in fixed point theory and some historical motivations, we refer the reader to [1]. Recall that a multi-valued mapping $\boldsymbol{T}: X \rightarrow \mathcal{B C}(X)$ is

- nonexpansive if $\mathcal{H}(\boldsymbol{T} x, \boldsymbol{T} y) \leq d(x, y)$ for all $x, y \in X$;

- Suzuki's generalized nonexpansive ([12]) if for all $x, y \in X$ the following implication holds:

$$
\frac{1}{2} \operatorname{dist}(x, \boldsymbol{T} x) \leq d(x, y) \Longrightarrow \mathcal{H}(\boldsymbol{T} x, \boldsymbol{T} y) \leq d(x, y)
$$

- quasi-nonexpansive if $\operatorname{Fix}(\boldsymbol{T}):=\{x \in X: x \in \boldsymbol{T} x\} \neq \varnothing$ and $\mathcal{H}(\boldsymbol{T} x, \boldsymbol{T} q) \leq d(x, q)$ for all $x \in X$ and $q \in \operatorname{Fix}(T)$

- quasi-nonexpansive ${ }^{*}$ if $\operatorname{SFix}(\boldsymbol{T}):=\{x \in X:\{x\}=\boldsymbol{T} x\} \neq \varnothing$ and $\mathcal{H}(\boldsymbol{T} x, \boldsymbol{T} q) \leq$ $d(x, q)$ for all $x \in X$ and $q \in \operatorname{SFix}(\boldsymbol{T})$.

Proposition 2.1. [12] Let $X:=(X, d)$ be a metric space and $\boldsymbol{T}: X \rightarrow \mathcal{B C}(X)$ be a multi-valued mapping. Then the following statements hold.

(a) If $\boldsymbol{T}$ is nonexpansive, then $\boldsymbol{T}$ is Suzuki's generalized nonexpansive.

(b) If $\boldsymbol{T}$ is Suzuki's generalized nonexpansive and $\operatorname{Fix}(\boldsymbol{T}) \neq \varnothing$, then $\boldsymbol{T}$ is quasi-nonexpansive.

(c) If $\boldsymbol{T}$ is quasi-nonexpansive and $\operatorname{SFix}(\boldsymbol{T}) \neq \varnothing$, then $\boldsymbol{T}$ is quasi-nonexpansive*.

Recall that $\boldsymbol{T}: X \rightarrow \mathcal{B C}(X)$ satisfies Condition $\left(E_{\mu}\right)$ where $\mu \geq 1[7,6]$ if $\operatorname{dist}(x, \boldsymbol{T} y) \leq$ $\mu \operatorname{dist}(x, \boldsymbol{T} x)+d(x, y)$ for all $x, y \in X$. Kudtha and Panyanak (see [11, Lemma 2.6(iii)]) claimed that every Suzuki's generalized nonexpansive mapping satisfies Condition $\left(E_{3}\right)$ by referring to Lemma 3.2 of Espínola et al. ([6]). However, the definitions of Suzuki's generalized nonexpansive mapping of [11] and that of [6] are not the same.

We now give a proof of this claim. We note that our multi-valued mapping $\boldsymbol{T}$ takes bounded and closed value rather than compact value as it was the case in [11].

Proposition 2.2. Suppose that $X$ is a metric space. If $\boldsymbol{T}: X \rightarrow \mathcal{B C}(X)$ is Suzuki's generalized nonexpansive, then it satisfies Condition $\left(E_{3}\right)$.

Proof. Let $x, y \in X$. We consider the following two cases.

Case 1: $\frac{1}{2} \operatorname{dist}(x, \boldsymbol{T} x) \leq d(x, y)$. It follows that

$$
\begin{aligned}
\operatorname{dist}(x, \boldsymbol{T} y) & \leq \operatorname{dist}(x, \boldsymbol{T} x)+\mathcal{H}(\boldsymbol{T} x, \boldsymbol{T} y) \\
& \leq \operatorname{dist}(x, \boldsymbol{T} x)+d(x, y) .
\end{aligned}
$$

Case 2: $d(x, y)<\frac{1}{2} \operatorname{dist}(x, \boldsymbol{T} x)$. Let $0<\varepsilon<\frac{1}{2} \operatorname{dist}(x, \boldsymbol{T} x)-d(x, y)$. We choose $x^{\prime} \in \boldsymbol{T} x$ such that $d\left(x, x^{\prime}\right)<\operatorname{dist}(x, \boldsymbol{T} x)+2 \varepsilon$ and note that

$$
\begin{aligned}
\operatorname{dist}(x, \boldsymbol{T} x) & \leq d(x, y)+\operatorname{dist}(y, \boldsymbol{T} x) \\
& <\frac{1}{2} \operatorname{dist}(x, \boldsymbol{T} x)-\varepsilon+\operatorname{dist}(y, \boldsymbol{T} x) .
\end{aligned}
$$


Then $\frac{1}{2} \operatorname{dist}(x, \boldsymbol{T} x)<\operatorname{dist}(y, \boldsymbol{T} x)-\varepsilon \leq d\left(y, x^{\prime}\right)-\varepsilon$. Note that $\frac{1}{2} \operatorname{dist}\left(x^{\prime}, \boldsymbol{T} x\right)=0 \leq$ $d\left(x, x^{\prime}\right)$ and hence $\operatorname{dist}\left(x^{\prime}, \boldsymbol{T} x^{\prime}\right) \leq \mathcal{H}\left(\boldsymbol{T} x, \boldsymbol{T} x^{\prime}\right) \leq d\left(x, x^{\prime}\right)$. It follows that

$$
\begin{aligned}
\frac{1}{2} \operatorname{dist}\left(x^{\prime}, \boldsymbol{T} x^{\prime}\right) & \leq \frac{1}{2} d\left(x, x^{\prime}\right)<\frac{1}{2} \operatorname{dist}(x, \boldsymbol{T} x)+\varepsilon \\
& <d\left(y, x^{\prime}\right)-\varepsilon+\varepsilon=d\left(y, x^{\prime}\right) .
\end{aligned}
$$

Thus $\mathcal{H}\left(\boldsymbol{T} x^{\prime}, \boldsymbol{T} y\right) \leq d\left(y, x^{\prime}\right)$.

Hence

$$
\begin{aligned}
\operatorname{dist}(x, \boldsymbol{T} y) & \leq \operatorname{dist}(x, \boldsymbol{T} x)+\mathcal{H}\left(\boldsymbol{T} x, \boldsymbol{T} x^{\prime}\right)+\mathcal{H}\left(\boldsymbol{T} x^{\prime}, \boldsymbol{T} y\right) \\
& \leq \operatorname{dist}(x, \boldsymbol{T} x)+d\left(x, x^{\prime}\right)+d\left(x^{\prime}, y\right) \\
& \leq \operatorname{dist}(x, \boldsymbol{T} x)+2 d\left(x, x^{\prime}\right)+d(x, y) \\
& \leq 3 \operatorname{dist}(x, \boldsymbol{T} x)+4 \varepsilon+d(x, y) .
\end{aligned}
$$

Letting $\varepsilon \downarrow 0$ gives $\operatorname{dist}(x, \boldsymbol{T} y) \leq 3 \operatorname{dist}(x, \boldsymbol{T} x)+d(x, y)$.

This completes the proof.

Suppose that $X:=(X, d)$ is a metric space. We say that $\boldsymbol{T}: X \rightarrow \mathcal{B C}(X)$ is $\Delta$-demiclosed de $^{*}$ (strongly demiclosed ${ }^{*}$, resp.) if $q \in \operatorname{SFix}(\boldsymbol{T})$ whenever $\left\{x_{n}\right\}$ is a sequence in $X$ such that $x_{n} \stackrel{\Delta}{\rightarrow} q \in X\left(\lim _{n} x_{n}=q \in X\right.$, resp. $)$ and $\lim _{n} \mathcal{H}\left(\left\{x_{n}\right\}, \boldsymbol{T} x_{n}\right)=0$. It follows from the definition that if $\boldsymbol{T}$ is $\Delta$-demiclosed ${ }^{*}$, then it is strongly demiclosed ${ }^{*}$. The following result is very similar to [11, Theorem 3.1] but the proof given here is simpler. Moreover, the uniform convexity as was the case in [11, Theorem 3.1] is superfluous.

Theorem 2.7. Suppose that $X$ is a metric space. If $\boldsymbol{T}: X \rightarrow \mathcal{K}(X)$ is a Suzuki's generalized nonexpansive mapping, then it is $\Delta$-demiclosed*.

Proof. Suppose that $\left\{x_{n}\right\}$ is a sequence in $X$ such that $x_{n} \stackrel{\Delta}{\rightarrow} q$ for some $q \in X$ and $\lim _{n} \mathcal{H}\left(\left\{x_{n}\right\}, \boldsymbol{T} x_{n}\right)=0$. For each $n \geq 1$, since $\boldsymbol{T} q$ is compact, we can choose $q_{n} \in \boldsymbol{T} q$ such that $d\left(x_{n}, q_{n}\right)=\operatorname{dist}\left(x_{n}, \boldsymbol{T} q\right)$. Moreover, since $\boldsymbol{T} q$ is compact, there exists a subsequence $\left\{q_{n_{k}}\right\}$ of $\left\{q_{n}\right\}$ such that $\lim _{k} q_{n_{k}}=u$ for some $u \in \boldsymbol{T} q$. Since $\boldsymbol{T}$ is Suzuki's generalized nonexpansive, it follows from Proposition 2.2 that $\boldsymbol{T}$ satisfies Condition $\left(E_{3}\right)$, that is, $\operatorname{dist}\left(x_{n_{k}}, \boldsymbol{T} q\right) \leq 3 \operatorname{dist}\left(x_{n_{k}}, \boldsymbol{T} x_{n_{k}}\right)+d\left(x_{n_{k}}, q\right)$. This implies that

$$
\begin{aligned}
d\left(x_{n_{k}}, u\right) & \leq d\left(x_{n_{k}}, q_{n_{k}}\right)+d\left(q_{n_{k}}, u\right) \\
& =\operatorname{dist}\left(x_{n_{k}}, \boldsymbol{T} q\right)+d\left(q_{n_{k}}, u\right) \\
& \leq 3 \operatorname{dist}\left(x_{n_{k}}, \boldsymbol{T} x_{n_{k}}\right)+d\left(x_{n_{k}}, q\right)+d\left(q_{n_{k}}, u\right) \\
& \leq 3 \mathcal{H}\left(\left\{x_{n_{k}}\right\}, \boldsymbol{T} x_{n_{k}}\right)+d\left(x_{n_{k}}, q\right)+d\left(q_{n_{k}}, u\right) .
\end{aligned}
$$

It follows that $\lim \sup _{k} d\left(x_{n_{k}}, u\right) \leq \lim \sup _{k} d\left(x_{n_{k}}, q\right)$. Note that $x_{n_{k}} \stackrel{\Delta}{\rightarrow} q$. Hence $u=q$. This implies that $q \in \boldsymbol{T} q$. To show that $\boldsymbol{T} q=\{q\}$, let $v \in \boldsymbol{T} q$. Note that $\frac{1}{2} \operatorname{dist}(q, \boldsymbol{T} q)=0 \leq$ $d\left(q, x_{n}\right)$. Hence $\mathcal{H}\left(\boldsymbol{T} q, \boldsymbol{T} x_{n}\right) \leq d\left(q, x_{n}\right)$ because $\boldsymbol{T}$ is Suzuki's generalized nonexpansive. Then

$$
\begin{aligned}
d\left(x_{n}, v\right) & \leq \mathcal{H}\left(\left\{x_{n}\right\}, \boldsymbol{T} q\right) \\
& \leq \mathcal{H}\left(\left\{x_{n}\right\}, \boldsymbol{T} x_{n}\right)+\mathcal{H}\left(\boldsymbol{T} x_{n}, \boldsymbol{T} q\right) \\
& \leq \mathcal{H}\left(\left\{x_{n}\right\}, \boldsymbol{T} x_{n}\right)+d\left(x_{n}, q\right) .
\end{aligned}
$$

It follows that $\lim \sup _{n} d\left(x_{n}, v\right) \leq \lim \sup _{n} d\left(x_{n}, q\right)$. Since $x_{n} \stackrel{\Delta}{\rightarrow} q$, we have $v=q$. Therefore $\boldsymbol{T} q=\{q\}$, that is, $q \in \operatorname{SFix}(\boldsymbol{T})$.

Recall that a multi-valued mapping $\boldsymbol{T}: X \rightarrow \mathcal{K}(X)$ 
- satisfies Condition $I^{*}$ if $\operatorname{SFix}(\boldsymbol{T}) \neq \varnothing$ and there exists a nondecreasing function $h:[0, \infty) \rightarrow[0, \infty)$ such that $h$ vanishes only at zero and

$$
h(\operatorname{dist}(x, \operatorname{SFix}(\boldsymbol{T}))) \leq \mathcal{H}(\{x\}, \boldsymbol{T} x) \text { for all } x \in X ;
$$

- is semicompact* if every sequence $\left\{x_{n}\right\}$ in $X$ with $\lim _{n} \mathcal{H}\left(\left\{x_{n}\right\}, \boldsymbol{T} x_{n}\right)=0$ contains a convergent subsequence.

The two notions above were introduced in [19] as a multi-valued version of the corresponding conditions in Definition 2.1.

We present the following selection theorem for a quasi-nonexpansive* multi-valued mapping taking compact values.

Theorem 2.8. Suppose that $X$ is a metric space and $\boldsymbol{T}: X \rightarrow \mathcal{K}(X)$ is a quasi-nonexpansive* multi-valued mapping. Then $T$ admits a selection $T: X \rightarrow X$ such that $T$ is quasi-nonexpansive and $\operatorname{Fix}(T)=\operatorname{SFix}(\boldsymbol{T})$. Furthermore, if $\boldsymbol{T}$ is or satisfies $P^{*}$, then the selection $T$ of $\boldsymbol{T}$ above is or satisfies $P$ where $P \in\{\Delta$-demiclosed, strongly demiclosed, Condition $I$, semicompact $\}$. (The last assertion reads, for example: If $\boldsymbol{T}$ is $\Delta$-demiclosed ${ }^{*}$, then $T$ is $\Delta$-demiclosed.)

Proof. Let $x \in X$. Note that the function $y \mapsto d(x, y)$ is continuous on the compact domain $\boldsymbol{T} x$. Hence there exists $x^{\prime} \in \boldsymbol{T} x$ such that $d\left(x, x^{\prime}\right)=\sup \{d(x, y): y \in \boldsymbol{T} x\}=\mathcal{H}(\{x\}, \boldsymbol{T} x)$. In particular, we define a selection $T: X \rightarrow X$ by $T x:=x^{\prime}$. Hence $d(x, T x)=\mathcal{H}(\{x\}, \boldsymbol{T} x)$ for all $x \in X$. It follows that $\operatorname{Fix}(T)=\operatorname{SFix}(\boldsymbol{T})$. Finally, we prove that $T$ is quasinonexpansive. To see this, let $x \in X$ and $q \in \operatorname{Fix}(T)(=\operatorname{SFix}(\boldsymbol{T}))$. It follows that $d(T x, q) \leq \mathcal{H}(\boldsymbol{T} x,\{q\})=\mathcal{H}(\boldsymbol{T} x, \boldsymbol{T} q) \leq d(x, q)$. This completes the proof of the first assertion. We note that the 'Furthermore' is a consequence of the following expression:

$$
d(x, T x)=\mathcal{H}(\{x\}, \boldsymbol{T} x) \text { for all } x \in X .
$$

The verification of the assertion is straightforward.

Corollary 2.1. Suppose that $X$ is a metric space $X$. If $\boldsymbol{T}: X \rightarrow \mathcal{K}(X)$ is Suzuki's generalized nonexpansive such that $\operatorname{SFix}(\boldsymbol{T}) \neq \varnothing$, then $\boldsymbol{T}$ admits a selection $T: X \rightarrow X$ such that $T$ is quasi-nonexpansive such that $\operatorname{Fix}(T)=\operatorname{SFix}(T)$ and $T$ is $\Delta$-demiclosed.

Using our result, we obtain the following convergence theorem for multi-valued mappings.

Theorem 2.9. Suppose that $X:=(X, d)$ is a complete $p$-uniformly convex metric space. Suppose that $\boldsymbol{T}: X \rightarrow \mathcal{K}(X)$ is quasi-nonexpansive*. Suppose that $\left\{\alpha_{n}\right\}$ and $\left\{\beta_{n}\right\}$ are two sequences in $[0,1]$ and suppose that $\left\{z_{n}\right\} \subset X$ is iteratively defined as follows: $z_{1} \in X$ is arbitrarily chosen and for each $n \geq 1$

$$
\begin{aligned}
& z_{n}^{\prime} \in \boldsymbol{T} z_{n} \text { such that } d\left(z_{n}, z_{n}^{\prime}\right)=\sup \left\{d\left(z_{n}, z\right): z \in \boldsymbol{T} z_{n}\right\} \\
& w_{n}:=\left(1-\beta_{n}\right) z_{n} \oplus \beta_{n} z_{n}^{\prime} ; \\
& w_{n}^{\prime} \in \boldsymbol{T} w_{n} \text { such that } d\left(w_{n}, w_{n}^{\prime}\right)=\sup \left\{d\left(w_{n}, w\right): w \in \boldsymbol{T} w_{n}\right\} \\
& z_{n+1}:=\left(1-\alpha_{n}\right) z_{n} \oplus \alpha_{n} w_{n}^{\prime} .
\end{aligned}
$$

(a) Suppose that $\boldsymbol{T}$ is $\Delta$-demiclosed ${ }^{*}$ and one of the following conditions holds:

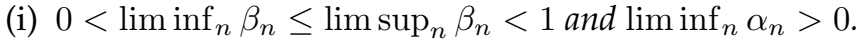

(ii) $\beta_{n}=0$ for all $n \geq 1$ and $0<\liminf _{n} \alpha_{n} \leq \lim \sup _{n} \alpha_{n}<1$.

Then $z_{n} \stackrel{\Delta}{\rightarrow} q \in \operatorname{SFix}(\boldsymbol{T})$ and $q=\lim _{n} \operatorname{Proj}_{\operatorname{SFix}(\boldsymbol{T})} x_{n}$.

(b) Suppose that one of the following conditions holds:

(i') $\boldsymbol{T}$ satisfies Condition $I^{*}$;

(ii') $\boldsymbol{T}$ is semicompact* and strongly demiclosed*.

Suppose that one of the following conditions holds: 
(i) $\sum_{n} \alpha_{n} \beta_{n}\left(1-\beta_{n}\right)=\infty$;

(ii) $\beta_{n}=0$ for all $n \geq 1$ and $\sum_{n} \alpha_{n}\left(1-\alpha_{n}\right)=\infty$.

Then $\left\{z_{n}\right\}$ converges strongly to a strict fixed point of $\boldsymbol{T}$.

Remark 2.1. Our Theorem 2.9 improves and extends Theorem LP in the following ways.

- Theorem 2.9 deals with a wider class of mappings than Theorem LP. In fact, it is easy to see that every Suzuki's generalized nonexpansive mapping with a strict fixed point is quasi-nonexpansive*.

- The assumptions on the parameters $\left\{\alpha_{n}\right\}$ and $\left\{\beta_{n}\right\}$ in our Theorem 2.9 are more general than the ones in Theorem LP. It is clear that the assumptions $\sum_{n} \alpha_{n} \beta_{n}=$ $\infty$ and $\lim _{n} \beta_{n}=0$ imply Condition (i) of Theorem 2.9(b).

- Our Theorem 2.9 includes a simple situation of the Mann's iteration and Kransnoselskii's iteration while it is not the case for Theorem LP. In fact, Theorem LP does not allow the case $\beta_{n}=0$ for all $n \geq 1$.

We now end the paper by showing that a class of mappings in Theorem 2.9 includes ones which are not Suzuki's generalized nonexpansive. Hence our result is a genuine generalization of Theorem LP.

Suppose that $X:=(X, d)$ is a metric space. Recall that $\boldsymbol{T}: X \rightarrow \mathcal{B C}(X)$ is

- Kannan's nonexpansive [23] if $\mathcal{H}(\boldsymbol{T} x, \boldsymbol{T} y) \leq \frac{1}{2} \operatorname{dist}(x, \boldsymbol{T} x)+\frac{1}{2} \operatorname{dist}(y, \boldsymbol{T} y)$ for all $x, y \in$ $X$;

- Chatterjea's nonexpansive if $\mathcal{H}(\boldsymbol{T} x, \boldsymbol{T} y) \leq \frac{1}{2} \operatorname{dist}(x, \boldsymbol{T} y)+\frac{1}{2} \operatorname{dist}(y, \boldsymbol{T} x)$ for all $x, y \in$ $X$.

We note that the definition of Chatterjea's nonexpansive mappings is introduced first time here and it is inspired by the work of Chatterjea [3]. For a single-valued mapping $T: X \rightarrow$ $X$, we say that $T$ is Kannan's nonexpansive (Chatterjea's nonexpansive, resp.) if $\boldsymbol{T}$ is Kannan's nonexpansive (Chatterjea's nonexpansive, resp.) where $\boldsymbol{T} x:=\{T x\}$ for all $x \in X$.

Proposition 2.3. Every Kannan's nonexpansive (Chatterjea's nonexpansive, resp.) mapping with a fixed point is quasi-nonexpansive. In particular, every Kannan's nonexpansive (Chatterjea's nonexpansive, resp.) mapping with a strict fixed point is quasi-nonexpansive*.

Proof. The proof of the assertion for Chatterjea's nonexpansive mappings is almost similar to the proof of that for Kannan's nonexpansive mappings, so it is omitted. Suppose that $\boldsymbol{T}: X \rightarrow \mathcal{B C}(X)$ is Kannan's nonexpansive with a fixed point. To see that $\boldsymbol{T}$ is quasinonexpansive, let $x \in X$ and $p \in \operatorname{Fix}(T)$. It follows that

$$
\begin{aligned}
\mathcal{H}(\boldsymbol{T} x, \boldsymbol{T} p) & \leq \frac{1}{2} \operatorname{dist}(x, \boldsymbol{T} x)+\frac{1}{2} \operatorname{dist}(p, \boldsymbol{T} p) \\
& =\frac{1}{2} \operatorname{dist}(x, \boldsymbol{T} x) \\
& \leq \frac{1}{2} d(x, p)+\frac{1}{2} \operatorname{dist}(p, \boldsymbol{T} x) \\
& \leq \frac{1}{2} d(x, p)+\frac{1}{2} \mathcal{H}(\boldsymbol{T} p, \boldsymbol{T} x) .
\end{aligned}
$$

This implies that $\mathcal{H}(\boldsymbol{T} x, \boldsymbol{T} p) \leq d(x, p)$.

Proposition 2.4. Every Kannan's nonexpansive (Chatterjea's nonexpansive, resp.) mapping taking compact values is $\Delta$-demiclosed*.

Proof. The proof of the assertion for Chatterjea's nonexpansive mappings is almost similar to the proof of that for Kannan's nonexpansive mappings, so it is omitted. Suppose that $\boldsymbol{T}: X \rightarrow \mathcal{K}(X)$ is Kannan's nonexpansive. We prove that $\boldsymbol{T}$ is $\Delta$-demiclosed ${ }^{*}$. Suppose 
that $\left\{x_{n}\right\}$ is a sequence in $X$ such that $x_{n} \stackrel{\Delta}{\rightarrow} q$ for some $q \in X$ and $\lim _{n} \mathcal{H}\left(\left\{x_{n}\right\}, \boldsymbol{T} x_{n}\right)=0$. For each $n \geq 1$, let $q_{n} \in \boldsymbol{T} q$ such that $d\left(x_{n}, q_{n}\right)=\mathcal{H}\left(\left\{x_{n}\right\}, \boldsymbol{T} q\right)$. (Such an element exists because of the compactness of $\boldsymbol{T} q$.) In particular, there exists a subsequence $\left\{q_{n_{k}}\right\}$ of $\left\{q_{n}\right\}$ such that $\lim _{k} q_{n_{k}}=u$ for some $u \in \boldsymbol{T} q$. Note that

$$
\begin{aligned}
\mathcal{H}\left(\boldsymbol{T} x_{n_{k}}, \boldsymbol{T} q\right) & \leq \frac{1}{2} \operatorname{dist}\left(x_{n_{k}}, \boldsymbol{T} x_{n_{k}}\right)+\frac{1}{2} \operatorname{dist}(q, \boldsymbol{T} q) \\
& \leq \frac{1}{2} \mathcal{H}\left(\left\{x_{n_{k}}\right\}, \boldsymbol{T} x_{n_{k}}\right)+\frac{1}{2} \mathcal{H}(\{q\}, \boldsymbol{T} q) \\
& \leq \frac{1}{2} \mathcal{H}\left(\left\{x_{n_{k}}\right\}, \boldsymbol{T} x_{n_{k}}\right)+\frac{1}{2} d\left(x_{n_{k}}, q\right)+\frac{1}{2} \mathcal{H}\left(\left\{x_{n_{k}}\right\}, \boldsymbol{T} x_{n_{k}}\right)+\frac{1}{2} \mathcal{H}\left(\boldsymbol{T} x_{n_{k}}, \boldsymbol{T} q\right) .
\end{aligned}
$$

This implies that

$$
\limsup _{k} \mathcal{H}\left(\boldsymbol{T} x_{n_{k}}, \boldsymbol{T} q\right) \leq \underset{k}{\lim \sup } d\left(x_{n_{k}}, q\right)
$$

Hence

$$
\begin{aligned}
& \limsup _{k} d\left(x_{n_{k}}, u\right)=\limsup _{k} d\left(x_{n_{k}}, q_{n_{k}}\right) \\
& =\limsup _{k} \mathcal{H}\left(\left\{x_{n_{k}}\right\}, \boldsymbol{T} q\right) \\
& \leq \limsup _{k}\left(\mathcal{H}\left(\left\{x_{n_{k}}\right\}, \boldsymbol{T} x_{n_{k}}\right)+\mathcal{H}\left(\boldsymbol{T} x_{n_{k}}, \boldsymbol{T} q\right)\right) \\
& \leq \limsup d\left(x_{n_{k}}, q\right) \text {. }
\end{aligned}
$$

Since $x_{n_{k}} \stackrel{\Delta}{\rightarrow} q$, we have $u=q$, that is, $q \in \boldsymbol{T} q$. To show that $q \in \operatorname{SFix}(\boldsymbol{T})$, let $v \in \boldsymbol{T} q$. It follows since $\boldsymbol{T}$ is quasi-nonexpansive (see Proposition 2.3) and $q \in \operatorname{Fix}(\boldsymbol{T})$ that

$$
\begin{aligned}
d\left(x_{n}, v\right) & \leq \mathcal{H}\left(\left\{x_{n}\right\}, \boldsymbol{T} q\right) \\
& \leq \mathcal{H}\left(\left\{x_{n}\right\}, \boldsymbol{T} x_{n}\right)+\mathcal{H}\left(\boldsymbol{T} x_{n}, \boldsymbol{T} q\right) \\
& \leq \mathcal{H}\left(\left\{x_{n}\right\}, \boldsymbol{T} x_{n}\right)+d\left(x_{n}, q\right) .
\end{aligned}
$$

In particular,

$$
\limsup _{n} d\left(x_{n}, v\right) \leq \limsup _{n} d\left(x_{n}, q\right) .
$$

Since $x_{n} \stackrel{\Delta}{\rightarrow} q$, we have $v=q$. This implies that $\boldsymbol{T} q=\{q\}$, that is, $q \in \operatorname{SFix}(\boldsymbol{T})$.

The following example shows that there exists a Kannan's nonexpansive mapping which is not Suzuki's generalized nonexpansive.

Example 2.1. Let $X:=[0,1]$ with the usual metric $d(x, y):=|x-y|$ for all $x, y \in X$. It is clear that $X$ is a complete 2-uniformly convex geodesic metric space. Let $T: X \rightarrow X$ be defined by

$$
T x:= \begin{cases}1-x & \text { if } x \in[0,1 / 3) \\ (x+1) / 3 & \text { if } x \in[1 / 3,1] .\end{cases}
$$

It follows from [25] that $T$ is Kannan's nonexpansive. For the sake of completeness, we give a proof. Let $x, y \in X$. We consider the following three cases.

Case 1: $x, y \in[0,1 / 3)$. In this case, we have $d(T x, T y)=|x-y| \leq 1 / 3<1-(x+y)=$ $\frac{1}{2}(1-2 x)+\frac{1}{2}(1-2 y)=\frac{1}{2} d(x, T x)+\frac{1}{2} d(y, T y)$.

Case 2: $x, y \in[1 / 3,1]$. It follows that $d(T x, T y)=\frac{1}{3}|x-y|=\frac{1}{6}|(2 x-1)-(2 y-1)| \leq$ $\frac{1}{6}|2 x-1|+\frac{1}{6}|2 y-1|=\frac{1}{2} d(x, T x)+\frac{1}{2} d(y, T y)$. 
Case 3: $x \in[0,1 / 3)$ and $y \in[1 / 3,1]$. Then $d(T x, T y)=\left|(1-x)-\frac{y+1}{3}\right| \leq \frac{1}{2}|1-2 x|+$ $\frac{1}{2}\left|\frac{2 y-1}{3}\right|=\frac{1}{2} d(x, T x)+\frac{1}{2} d(y, T y)$.

Next, we show that $T$ is not Suzuki's generalized nonexpansive. Choose $x=1 / 3$ and $y=5 / 18$. Then

but

$$
\frac{1}{2} d(x, T x)=\frac{1}{2}\left|\frac{1}{3}-\frac{4}{9}\right|=\frac{1}{18}=d(x, y)
$$

$$
d(T x, T y)=\left|\frac{4}{9}-\frac{13}{18}\right|=\frac{5}{18}>\frac{1}{18}=d(x, y) .
$$

Acknowledgement. The authors are grateful to the two referees for their comments and suggestions. The authors would like to thank Professor Pongsakorn Yotkaew for his comment and suggestion on the first version of the manuscript. The research of the second author was supported by the Thailand Research Fund and Khon Kaen University under grant RSA6280002.

\section{REFERENCES}

[1] Berinde, V.; Păcurar, M. The role of the Pompeiu-Hausdorff metric in fixed point theory. Creat. Math. Inform. 22 (2013), 143-150

[2] Browder, F. E. Semicontractive and semiaccretive nonlinear mappings in Banach spaces. Bull. Amer. Math. Soc. 74 (1968), 660-665

[3] Chatterjea, S. K. Fixed-point theorems. C. R. Acad. Bulgare Sci. 25 (1972), 727-73.

[4] Chidume, C. E.; Mutangadura, S. A. An example of the Mann iteration method for Lipschitz pseudocontractions. Proc. Amer. Math. Soc. 129 (2001), 2359-2363

[5] Clarkson, J. A. Uniformly convex spaces. Trans. Amer. Math. Soc. 40 (1936), no. 3, 369-414

[6] Espínola, R.; Lorenzo, P.; Nicolae, A. Fixed points, selections and common fixed points for nonexpansivetype mappings. J. Math. Anal. Appl. 382 (2011), no. 2, 503-515

[7] Garcia-Falset, J.; Lorens-Fuster, E.; Suzuki, T. Fixed point theory for a class of generalized nonexpansive mappings. J. Math. Anal. Appl. 375 (2011), 185-195

[8] Goebel, K.; Kirk, W. A. Topics in metric fixed point theory. Cambridge Studies in Advanced Mathematics, 28. Cambridge University Press, Cambridge, 1990.

[9] Ishikawa, S. Fixed points by a new iteration method. Proc. Amer. Math. Soc. 44 (1974), 147-150

[10] Kimura, Y.; Saejung, S.; Yotkaew, P. The Mann algorithm in a complete geodesic space with curvature bounded above. Fixed Point Theory Appl. (2013), 2013:336, 13 pp.

[11] Kudtha, A.; Panyanak, B. Common endpoints for Suzuki mappings in uniformly convex hyperbolic spaces. Thai J. Math. (2018), Special issue, 159-168

[12] Laokul, T.; Panyanak, B. A generalization of the $(C N)$ inequality and its applications. Carpathian J. Math. 36 (2020), no. 1, 81-90

[13] Lim, T. C. Remarks on some fixed point theorems. Proc. Amer. Math. Soc. 60 (1976), 179-182

[14] Mann, W. R. Mean value methods in iteration. Proc. Amer. Math. Soc. 4 (1953), 506-510

[15] Naor, A.; Silberman, L. Poincaré inequalities, embeddings, and wild groups. Compos. Math. 147 (2011), no. $5,1546-1572$

[16] Ohta, S. Convexities of metric spaces. Geom. Dedicata. 125 (2007), 225-250

[17] Opial, Z. Weak convergence of the sequence of successive approximations for nonexpansive mappings. Bull. Amer. Math. Soc. 73 (1967), 591-597

[18] Outlaw, C. L. Mean value iteration of nonexpansive mappings in a Banach space. Pacific J. Math. 30 (1969), 747-750

[19] Panyanak, B. Approximating endpoints of multi-valued nonexpansive mappings in Banach spaces. J. Fixed Point Theory Appl. 20 (2018), no. 2, Art. 77, 8 pp.

[20] Rus, I. A.; Petruşel, A.; Petruşel, G. Fixed point theory. Cluj University Press, Cluj-Napoca, 2008

[21] Saejung, S.; Suantai, S.; Yotkaew, P. A note on "Common fixed point of multistep Noor iteration with errors for a finite family of generalized asymptotically quasi-nonexpansive mappings". Abstr. Appl. Anal. (2009), Art. ID 283461, 9 pp.

[22] Senter, H. F.; Dotson, W. G. Approximating fixed points of nonexpansive mappings. Proc. Amer. Math. Soc. 44 (1974), 375-380

[23] Shiau, C.; Tan, K. K.; Wong, C. S. A class of quasi-nonexpansive multi-valued maps. Canad. Math. Bull. 18 (1975), 709-714 
[24] Takahashi, W.; Kim, G. E. Approximating fixed points of nonexpansive mappings in Banach spaces. Math. Japon. 48 (1998), 1-9

[25] Wong, C. S. On Kannan maps. Proc. Amer. Math. Soc. 47 (1975), 105-111

[26] Xu, H. K. Inequalities in Banach spaces with applications. Nonlinear Anal. 16 (1991), 1127-1138

DePARTMENT OF MATHEMATiCS

FACULTY OF SCIENCE

KHON KAEN University, KHON KAEN, 40002, ThaILAND

E-mail address: jenjira.puiwong@kkumail.com

DEPARTMENT OF MATHEMATICS

FACULTY OF SCIENCE

KHON KAEN UNIVERSITY

KHON KAEN, 40002, THAILAND

Research Center for Environmental and Hazardous Substance Management (EHSM)

KHON KAEN UNIVERSITY

KHON KAEN, 40002, THAILAND

E-mail address: saejung@kku.ac.th 